\title{
Linx
}

Revue des linguistes de l'université Paris X Nanterre

66-67| 2012

Dynamiques de la construction des sens attendus et inattendus dans les langues

\section{Sens inattendu ou attendu de la concession marquée par si et par même si}

\section{Maria Svensson}

\section{CpenEdition}

\section{Journals}

Édition électronique

URL : http://journals.openedition.org/linx/1451

DOI : $10.4000 / \operatorname{linx} .1451$

ISSN : 2118-9692

Éditeur

Presses universitaires de Paris Nanterre

Édition imprimée

Date de publication : 15 septembre 2012

Pagination : 83-98

ISSN : 0246-8743

Référence électronique

Maria Svensson, «Sens inattendu ou attendu de la concession marquée par si et par même si », Linx [En ligne], 66-67 | 2012, mis en ligne le 15 septembre 2013, consulté le 01 mai 2019. URL : http:// journals.openedition.org/linx/1451; DOI : 10.4000/linx.1451 


\title{
Sens inattendu ou attendu de la concession marquée par si et par même si
}

\author{
Maria Svensson \\ Université d'Uppsala
}

\section{Introduction}

La conjonction française si est surtout liée à une valeur hypothétique, conditionnelle, qui pourrait être considérée comme sa valeur attendue. Pourtant, il est bien connu qu'une combinaison de deux propositions à l'aide de si peut également véhiculer une valeur moins attendue, lorsque $s i$ introduit une proposition exprimant un contenu factuel (p) (Stage, 1991, Corminbœuf, 2009 et 2013, Monte, 2009, Provôt, 2011). Dans le cas où $p$ est présenté comme factuel, la relation entre $p$ et $q$ est parfois à interpréter comme concessive. La relation concessive consiste en un manque de réalisation d'une conclusion déductible de $p$, c'est-à-dire en une conclusion réfutée, décrite par Monte (2009, p. 112) de la manière suivante :

Dans les systèmes concessifs, la reprise de $\mathrm{P}$ dans la si $\mathrm{P}$ est suivie de l'assertion en $\mathrm{Q}$ d'un argument qui vient contrarier l'inférence que l'allocutaire aurait été en droit de former à partir du contenu de $\mathrm{P}$.

Ainsi, dans l'exemple 1, on aurait pu tirer de $p$, c'est-à-dire le fait que MarieLouise refuse un divorce, la conclusion que ce refus impliquerait aussi qu'elle s'oppose à une séparation, ce qui est réfuté par $q$. 
Exemple 1: Si elle refuse un divorce, Marie-Louise lui offre une séparation à l'amiable et confirme qu'elle ne reviendra jamais. (de Villepin, 2002, p. 386)

Cette valeur concessive peut être considérée comme inattendue par rapport à la relation la plus fréquemment actualisée dans les constructions avec si, la relation prédictive, comme celle de l'exemple 2 :

Exemple 2: Mais si l'on cesse de fumer avant 35 ans, on réduit beaucoup ce vieillissement précoce. (Tubiana, 2003, p. 172)

La relation prédictive (terme de Dancygier et Sweetser, 2005) d'une construction conditionnelle consiste dans le fait qu'elle "prédit la réalisation de l'état de choses $q$ » (Kronning, 2009a, p. 100), au moyen d'une relation causale ou de consécution $\mathrm{R}$ qui relie la vérification de $q$ à celle de $p$. Selon Kronning (2013), cette relation conditionnelle-prédictive est assertée par le locuteur ${ }^{1}$. Dans l'exemple 2, il y aurait une relation de consécution entre le fait de cesser de fumer avant 35 ans et une réduction $\mathrm{du}$ vieillissement, qui constituerait la base de la relation conditionnelle-prédictive assertée.

\section{Le caractère inattendu de l'emploi concessif de si par rapport à celui de même si}

L'emploi concessif de si peut être considéré comme inattendu, car la valeur «par défaut » des constructions avec si est premièrement conditionnelle-prédictive et deuxièmement non factuelle. Cet emploi de si est ainsi doublement inattendu, étant donné que ce n'est pas seulement la non-actualisation de la relation prédictive actualisée dans l'exemple 2 qui est inattendue, mais que aussi la valeur factuelle pourrait être considérée comme inattendue.

À commencer par la relation conditionnelle-prédictive, l'emploi concessif de si se caractérise par le fait qu'une telle relation prédictive n'est pas assertée, mais que c'est une relation concessive qui est actualisée lors de l'interprétation du lien entre $p$ et $q$. Certes, on pourrait dire que la relation prédictive est présente dans l'emploi concessif aussi, étant donné que la concession est basée sur une telle valeur prédictive, mais ce qui importe, c'est que l'effet $q$ attendu par la réalisation de $p$ n'est pas réalisé, et que la vérité de cette conclusion $q$, la conséquence attendue, est réfutée lors de la concession. Le fait que le locuteur n'asserte pas une telle relation conditionnelle-prédictive peut-être considéré comme non-attendu dans les cas où c'est la conjonction si qui est employée pour relier ces propositions de la relation concessive. De plus, la concession englobe en soi une valeur inattendue, par la réfutation d'une conclusion attendue liée à $p$.

Le deuxième aspect inattendu de cet emploi de si est le fait que $p$ est dans cet emploi présenté comme factuel, si l'on considère que si éveille des attentes d'un

\footnotetext{
${ }^{1}$ Par «locuteur», nous entendons en principe ce que Kronning (2013) ainsi que Nølke et al. (2004, p. 38) appellent le locuteur de l'énoncé, correspondant au locuteur en tant que tel chez Ducrot (1984, p. 199), être de discours qui, selon Kronning (2013, p. 103), « assume la responsabilité (modale) de l'énoncé ».
} 
contenu sémantique à suivre qui est non factuel, et par rapport auquel le locuteur indique des attitudes épistémiques différentes; positive ("potentiel neutre»), faiblement négative (" potentiel faible »), et fortement négative (《iréel» ou «contrefactuel») (Kronning, 2009a, p. 102). Dans l'exemple 2 par contre, la valeur de $p$ est non factuelle (hypothétique, même si l'attitude épistémique est dans ce cas probablement positive), valeur qui pourrait être considérée comme plus attendue dans le cas de si que la valeur factuelle ${ }^{2}$ actualisée dans l'exemple 1. C'est notamment le caractère inattendu de cette factualité qui rend cet emploi de si contradictoire dans un certain sens. Comme Monte (2009, p. 105) l'exprime à propos des emplois non conditionnels de si, il y a "une contradiction [...] entre le choix de si et les indications cotextuelles, contradiction créatrice de tension ».

En ce qui concerne la factualité, l'emploi concessif factuel de si se distingue de l'emploi de même si (l'exemple 3), où le fait exprimé par la subordonnée peut être non factuel aussi bien que factuel (Morel, 1996, p. 36 ; Mossberg, 2009, p. 183 ; Lindschouw, 2011, p. 266), et où l'interprétation concessive est également l'interprétation par défaut, et par conséquent, la plus attendue.

Exemple 3: Dans un tel contexte, la personne âgée, fragilisée, s'interroge sur ses capacités, et même si sa vie a été une réussite, elle se juge inutile, voire nuisible, a honte d'être encore là. (Tubiana, 2003, p. 343)

Dans l'exemple 3, $p$ [sa vie a été une réussite] est présenté comme quelque chose de non factuel (bien que potentiel), ce qui dans le cas de même si est plutôt attendu ${ }^{3}$. Comme la subordonnée est introduite par même si, aussi la valeur concessive de la relation présentée dans l'exemple 3 entre la réussite de la vie de quelqu'un et le fait de se sentir inutile et nuisible en tant qu'âgé, est plus attendue comparée à celle actualisée par l'emploi concessif de si.

Notre hypothèse est que la valeur de factualité et surtout celle de concession sont plus attendues dans le cas de même si que dans le cas de si, ce dont nous essaierons de trouver des indices. La factualité de cet emploi de si ainsi que sa valeur concessive sont aussi inattendues par rapport à la valeur épistémique et prédictive de son emploi conditionnel-prédictif. Nous proposons une analyse des différences entre même si et l'emploi concessif de si, ainsi que de leurs similarités par rapport à l'emploi conditionnel-prédictif de si.

\footnotetext{
${ }^{2}$ Dans les termes de Kronning (2009a ; 2009b), cette valeur correspondrait vraisemblablement à une attitude épistémique positive forte. La terminologie de Kronning étant conçue pour l'analyse de l'emploi conditionnel-prédictif de si, où l'attitude épistémique n'est en principe pas positive forte, elle ne distingue pas une attitude épistémique positive forte d'une attitude épistémique positive faible, distinction qui correspondrait à celle mise en place entre une attitude épistémique négative forte et une attitude épistémique négative faible.

${ }^{3}$ En ce qui concerne la fréquence des occurrences de même si relevant d'un emploi factuel ou non factuel, il semble y avoir certaines divergences entre les données de Lindschouw (2011, p. 266) et celles de Mossberg (2009, p. 190), mais il n’y a pas de doute que le contenu de la subordonnée introduite par même si peut être présenté soit comme factuel soit comme non factuel.
} 


\section{But de la comparaison}

Le but de la comparaison de l'emploi concessif de si et celui de même si, ainsi que l'emploi conditionnel-prédictif de si, est de constater des différences formelles entre ces trois emplois. L'objectif de cette comparaison des caractéristiques formelles d'occurrences de si en emploi concessif et de même si est de voir si des différences formelles éventuelles peuvent révéler des différences au niveau sémantique qui imposeraient certaines contraintes ou qui expliqueraient certaines tendances formelles de l'emploi de ces deux conjonctions. Le but de la comparaison des mêmes caractéristiques formelles de si en emploi concessif ainsi que de même si avec les occurrences analysées de l'emploi conditionnel-prédictif de si, est d'éventuellement pouvoir constater que certains de ces traits formels sont plutôt liés à la concession et à l'interprétation concessive en général.

Le but est aussi de montrer des indices du fait que la relation concessive est moins attendue dans le cas de si que dans les cas de même si. Nous supposons que certaines différences formelles, notamment concernant la tendance à ajouter un marqueur supplémentaire de la concession, pourraient être liées à des différences de valeur attendue ou inattendue de la relation concessive.

\section{III. Études antérieures de si en emploi concessif et de même si}

Nous basons l'analyse de si sur des études antérieures portant sur son emploi factuel, notamment celle de Stage (1991), qui propose une catégorisation des emplois factuels de si ainsi que certains critères de distinction de ces catégories, Corminbœuf (2013), à propos de la factualité de cet emploi, et Monte (2009), qui étudie surtout la valeur dialogique de si en emploi concessif et comparatif. En ce qui concerne la relation concessive, il faut mentionner Morel (1996), dont surtout la distinction de trois types de concession, la concession logique, la concession rectificative et la concession argumentative (Morel, 1996, p. 6-20) est intéressante pour une étude comparative de si concessif et même si, étant donné que si ne semble pouvoir apparaitre qu'en contexte de concession argumentative, tandis que même si, selon Morel (1996, p. 35), peut marquer les trois types de concession.

La conjonction même si a également été décrite par Morel (1996) et surtout par Piot (2004), quoique cette dernière mette en question notamment le statut de conjonction composée de même si (Piot, 2004, p. 190). Nous n'entrerons pas dans les détails de l'analyse du statut composé ou non de la configuration de même si, mais tenons à souligner que nous inclurons dans l'étude toutes les occurrences de même si de notre corpus, que celles-ci soient factuelles ou non. Par cette démarche, notre étude se distingue de celle de Lindschouw (2011) aussi bien que celle de Mossberg (2009), où ces cas sont traités séparément. Notre étude inclut aussi bien des cas où il pourrait plutôt s'agir d'une subordonnée conditionnelle-prédictive introduite par si, précédée par même, comme ce nous semble être le cas dans l'exemple 4, que d'une relation concessive factuelle. 
Exemple 4: M. Bergeret s'est enrichi grâce à la bienfaisance qui valorise le donateur, mais avilit le mendiant pour qui la pièce reçue, quelle qu'elle soit, n'a pas de valeur puisqu'il ne l'a obtenue qu'en exhibant son incapacité à la gagner autrement. Le pauvre reste pauvre, même síl peut dépenser l'argent d'autrui. (Smith, 2003, p. 111)

Faute de critères fiables et univoques permettant de distinguer les deux types de valeurs, il vaut mieux ne pas distinguer quantitativement les occurrences du type présenté dans l'exemple 4 des cas où $p$ est plutôt factuel, même si les marqueurs de factualité auxquels prend recours Kjellmer (1989, p. 261) pour identifier des occurrences d'interprétation factuelle de $p$ après even if and even though peuvent indiquer le statut factuel de certaines occurrences.

\section{Points de départ théoriques polyphoniques}

Dans notre analyse, nous nous inspirons de Kronning (notamment 2009a; 2009 b ; 2013 ; à paraître) et de sa distinction des attitudes épistémiques, ainsi que de sa théorie modale de la polyphonie, la TMP, qu'il a appliquée notamment à l'analyse de $s i$, particulièrement dans son emploi conditionnel-prédictif.

Une théorie polyphonique nous semble indispensable pour traiter la relation concessive. Une chose qui distingue peut-être l'emploi concessif de si de son emploi conditionnel-prédictif est le statut polyphonique de $p$. Dans l'emploi concessif de $s i$, c'est toujours la relation concessive argumentative qui est actualisée. Cette relation concessive argumentative semble se caractériser par rapport à la concession rectificative entre autre par la valeur concédée de $p$; le locuteur de l'énoncé ne prend pas en charge la vérité de $p$, mais il la concède (Morel, 1996, p. 19-20). Pour Morel, un énoncé concédé est un «énoncé dont la validation provient d'un support énonciatif différent de celui de l'autre proposition, auquel l'énonciateur donne néanmoins son assentiment» (1996, p. 148), tandis qu'un énoncé asserté est un "énoncé dont l'énonciateur prend en charge la validation » (1996, p. 147).

Kronning exprime la valeur polyphonique de la construction concessive certes $P$, mais $Q$, qui actualise également la concession argumentative, dans le cadre de sa théorie modale de la polyphonie (Kronning, 2013, p. 103) de la manière suivante :

Si un être de discours est associé à une instance discursive, cette instance [discursive] est déjà identifiée à un autre être de discours. Ainsi, dans une construction concessive telle que Certes $P$, mais $Q$, l'ID [instance discursive] de $P$ est déjà assignée par identification (typiquement) à l'allocutaire, avant d'être attribuée par association au locuteur de l'énoncé. Nous dirons que le locuteur (LOC) «accorde », dans ce cas, le point de vue $P$.

Il nous semble que la structure polyphonique de constructions concessives avec si, ainsi que des cas où même si apparaît dans une concession argumentative, devrait pouvoir être décrite de la même manière, et cette description semble, du moins au 
niveau superficiel, être compatible avec l'analyse de Morel et sa distinction entre valeur assertée ou concédée de $p$.

Puisque la relation concessive est toujours argumentative avec si (Morel, 1996, p. 35 ; Svensson, 2013, p. 343), il semble que $p$ soit toujours concédé, ou " accordé », lors de l'emploi concessif de si. Par contre, selon Morel, dans l'emploi de même si, $p$ n’est pas nécessairement concédé, mais il serait parfois asserté, car cette conjonction peut apparaitre dans des contextes de tous les trois types de relation concessive proposés par Morel (1996, p. 6-20). La relation concessive marquée par même si peut être logique (l'exemple 5), argumentative (l'exemple 6) ou rectificative (l'exemple 7) (Morel, 1996, p. 35).

Exemple 5: Il faut leur être fidèle et participer aux réunions, même si l'on est fatigué, sans quoi les liens se distendent vite. (Tubiana, 2003, p. 377)

Exemple 6: Cela fait se transformer le regard porté sur les femmes. Les hommes voient en elles, beaucoup moins passives, de vraies associées avec lesquelles ils doivent compter et qui, même s'ils sont bien loin de les considérer comme leurs égales, méritent du moins d'être traitées par eux, pucelles ou dames, selon les règles. (Duby, 1995, p. 183)

Exemple 7: Mais, comme le champ politique et le champ économique, et beaucoup plus que le champ scientifique, artistique ou littéraire ou même juridique, le champ journalistique est soumis en permanence à l'épreuve des verdicts du marché, à travers la sanction, directe, de la clientèle ou, indirecte, de l'audimat (même si l'aide de l'État peut assurer une certaine indépendance à l'égard des contraintes immédiates du marché). (Bourdieu, 1996, p. 84-85)

Dans la relation rectificative (l'exemple 7), $p$ est selon Morel (1996, p. 20) asserté par le locuteur, qui prend en charge ce point de vue, aussi bien que celui de $q$. La possibilité d'emploi rectificatif ouvre donc la possibilité de même si d'introduire une subordonnée exprimant un contenu asserté, si l'on suit Morel. Nous n'entrerons pas ici dans les détails des critères définitoires de l'assertion, ni dans ceux de la subordination, mais nous ne pouvons renoncer à mettre en doute le statut syntaxique de ce qui suit même si dans ce cas, jugeant insatisfaisant un modèle dans lequel le statut de la subordination est indépendant de la valeur énonciative. Il nous semble qu'il faudrait examiner la possibilité de subordonnées de véhiculer une assertion; s'il est possible d'asserter un contenu dans une proposition subordonnée, l'assertion est vraisemblablement moins forte ou du moins différente que celle qui est faite sous forme d'une proposition principale. Renvoyant à Kronning (1992) à ce propos, nous nous contentons de constater que les critères de définition de l'assertion, et surtout son lien avec la définition de la subordination syntaxique, mériteraient une étude plus approfondie que celles qui à notre connaissance ont été accomplies, mais qu'il semble sans aucun doute $y$ avoir une différence dans la valeur énonciative, voire polyphonique, entre les trois types de concession. 


\section{Méthode}

Notre analyse de si et de même si se base sur des données fournies par le corpus C-ParaFraS-HumSam, composé de textes de littérature spécialisée en sciences humaines, publiés entre 1995 et 2005, dont les textes français comportent 1,98 millions de mots, décrit notamment dans Svensson (2010, p. 49-57).

Par la recherche de si ou de s' dans ce corpus, 4500 occurrences de si ont été relevées à l'aide de Wordsmith Tools. Après cette première recherche des occurrences de si, et après avoir écarté tous les cas de si adverbe intensifiant ainsi que ceux de si introduisant une subordonnée interrogative, a été effectuée une séparation des occurrences analysées comme relevant de l'emploi conditionnel-prédictif de celles relevant d'un emploi factuel, desquelles ont ensuite été écartées celles que nous avons analysées comme actualisant une valeur concessive. Lors de cette analyse, nous avons pu identifier les occurrences où si est précédé de même pour former la conjonction même si.

Ayant auparavant (voir par exemple Svensson 2013 ; 2012) renoncé à distinguer quantitativement les données concessives des données adversatives de si, nous avons cette fois-ci fait un choix des occurrences nous semblant vraisemblablement relever d'un emploi concessif, pour permettre une analyse quantitative comparant les caractéristiques formelles avec celles des occurrences de même si, qui ne semble guère pouvoir actualiser une valeur adversative ${ }^{4}$.

Évidemment, il y a des risques que parmi les occurrences exclues, jugées relever d'un emploi plutôt adversatif, figurent certaines occurrences qui selon d'autres critères seraient considérées comme concessives. Consciente de ce risque, nous avons néanmoins tenu à concentrer cette étude sur le cas concessif. Il importe quand même de soulever le risque de subjectivité, et également de mentionner que parmi les occurrences que nous avons traitées comme concessives, certaines, approximativement une trentaine, se trouvent à la limite entre la concession et le contraste, comme celle dans l'exemple 8 :

Exemple 8: Si, au Moyen Âge, les façades des cathédrales comme celles des maisons étaient peintes dans les teintes les plus vives, la couleur n'avait pas eu sa place dans les intérieurs modestes. (Beaucarnot, 2002, p. 374)

Dans l'exemple 8, il y a un contraste entre la présence et l'absence de couleurs ainsi que entre les façades des cathédrales et les intérieurs modestes, ce qui indiquerait qu'il s'agit d'un emploi adversatif. En même temps, la présence de couleurs, de «teintes les plus vives » à l'extérieur, aux façades, pourrait mener à la conclusion que les intérieurs modestes seraient également peints en couleurs vives, ce qui est refusé dans q. La possibilité d'interprétation d'une inférence réfutée indique qu'il y a également une valeur concessive qui est actualisée par cette séquence.

Ayant jugé que la valeur concessive, même si le lien inférentiel entre $p$ et la conclusion réfutée est basée sur un contraste lexical ou formel, est primordiale pour l'interprétation du lien entre $p$ et $q$ dans ces occurrences, nous avons opté pour le choix de les inclure dans le corpus analysé. Il semble que nous suivions par cette

\footnotetext{
${ }^{4}$ Notre analyse se base sur les définitions de la relation concessive et de la relation adversative de Gettrup \& Nølke (1984, p. 5-7) et leur développement chez Mossberg (2006, p. 33-36), ainsi que les tests de distinction des emplois factuels de si proposés par Stage (1991, p. 178).
} 
option la démarche de Monte (2009, p. 103), qui affirme certes que «des systèmes concessifs aux systèmes comparatifs à valeur oppositive, il y a un continuum plus qu'une coupure franche", mais qui distingue toutefois quantitativement les occurrences de si concessive (inférentiel) et celles de si adversatif-additif (comparatif) parmi ses occurrences de si.

Les occurrences de si que nous avons finalement considérées comme relevant d'un emploi concessif et que nous avons incluses dans l'étude s'élèvent à un nombre de 390. Nous avons relevé 434 occurrences de même si dans le corpus. Nous avons également analysé 400 occurrences de si en emploi conditionnel-prédictif pour comparer celles-ci avec celles de même si et de si en emploi concessif en ce qui concerne certaines caractéristiques formelles.

\section{Différence entre si et même si au niveau de l'attitude épistémique de $p$}

Comme déjà évoqué, $p$ est présenté comme factuel lors de l'emploi concessif de si, comme dans l'exemple 9 :

Exemple 9: Pierre Laval a toujours détesté la guerre. Si, avec une apparente maestria, il a glissé de l'extrême gauche à la droite la plus conservatrice, il n'a jamais répudié l'idéal de sa jeunesse : le pacifisme. (Decaux, 1996, p. 295)

Avec même si, $p$ peut être factuel ou non factuel (Morel, 1996, page 36 ; Mossberg, 2009, p. 183 ; Lindschouw, 2011, p. 266). Nos données confirment ces différences constatées auparavant entre ces deux marqueurs. Dans l'exemple 10, $p$ est présenté comme non factuel, tandis que dans l'exemple 11, $p$ est factuel.

Exemple 10: Il faut leur être fidèle et participer aux réunions, même si l'on est fatigué, sans quoi les liens se distendent vite. (Tubiana, 2003, p. 377)

Exemple 11: Cela fait se transformer le regard porté sur les femmes. Les hommes voient en elles, beaucoup moins passives, de vraies associées avec lesquelles ils doivent compter et qui, même síls sont bien loin de les considérer comme leurs égales, méritent du moins d'être traitées par eux, pucelles ou dames, selon les règles. (Duby, 1995, p. 183)

Étant donné que même si peut introduire une subordonnée exprimant un $p$ soit factuel soit non factuel, tandis que la concession actualisée par si implique toujours un $p$ factuel, cette valeur factuelle de l'emploi concessif de si peut être considérée comme plus attendue que celle d'un $p$ introduit par même si. Par contre, par rapport à l'emploi, plus fréquent, conditionnel-prédictif de si, cette valeur épistémique de $p$ est plutôt inattendue.

\section{Comparaison formelle entre si et même si}

Parmi les traits formels analysés, il y a certains qui ne permettent pas de distinguer l'emploi concessif de si de celui de même si, ni guère de l'emploi 
conditionnel-prédictif de si. Ceci est le cas notamment des temps verbaux, où aussi bien l'emploi concessif de si que l'emploi de même si se caractérisent par l'emploi de temps verbaux identiques dans $\mathrm{P}$ et $\mathrm{Q}$. Ce temps verbal est le plus souvent celui du présent de l'indicatif. La présence de la négation dans Q est un peu plus fréquente dans le cas de même si que dans le cas de l'emploi concessif de si, mais elle est tout aussi fréquente dans l'emploi conditionnel-prédictif de si. Par contre, en ce qui concerne la position de $\mathrm{P}$ par rapport à $\mathrm{Q}$, nous avons pu constater des différences entre les marqueurs, et également dans le marquage supplémentaire dans $\mathrm{Q}$ de la relation concessive.

\section{Temps verbaux}

C'est surtout l'emploi des temps verbaux identiques, et dans une certaine mesure, l'emploi du présent de l'indicatif, qui est plus fréquent dans les occurrences relevées de l'emploi concessif de si ainsi que dans celles de même si que dans les 400 occurrences relevées de l'emploi conditionnel-prédictif. Dans plus que trois quarts des cas $(82 \%$ des 390 occurrences de si concessif et $76 \%$ des 434 occurrences de même si), le même temps verbal est employé dans Q que comme dans $\mathrm{P}$, ce qui distingue ces occurrences des 400 occurrences analysées de l'emploi conditionnel-prédictif de $s i$, où tel est le cas dans $47 \%$ des occurrences seulement. Aussi bien avec même si dans l'exemple 12, reprenant l'exemple 4, que dans l'exemple 13 avec si en emploi concessif, c'est le présent de l'indicatif qui est employé dans $\mathrm{P}$ et dans Q :

Exemple 12: M. Bergeret s'est enrichi grâce à la bienfaisance qui valorise le donateur, mais avilit le mendiant pour qui la pièce reçue, quelle qu'elle soit, n'a pas de valeur puisqu'il ne l'a obtenue qu'en exhibant son incapacité à la gagner autrement. Le pauvre reste pauvre, même sill peut dépenser l'argent d'autrui. (Smith, 2003, p. 111)

Exemple 13: Si le corps « libéré » des publicités est souvent celui des femmes, en l'occurrence, il s'agit souvent de corps libérés de ses vêtements. (Détrez, 2002, p. 187)

Dans trois quarts des cas de $s i$ en emploi concessif ( $79 \%$ des occurrences de $s i$ en emploi concessif) aussi bien que de même si (78\% des occurrences), c'est le présent de l'indicatif qui apparait dans P. Ceci est aussi le cas le plus fréquent pour les occurrences analysées de si en emploi conditionnel-prédictif, mais alors c'est dans $66 \%$ des cas. La ressemblance sur ces points entre l'emploi concessif de si est les occurrences de même si indique que ces caractéristiques comparées sont plutôt liées à la valeur concessive qu'à l'emploi concessif de si seul.

\section{Négation dans $Q$}

Nous avons analysé la fréquence d'occurrences avec négation dans $\mathrm{P}$ ou $\mathrm{Q}$, surtout parce que la négation dans $Q$ a été présentée par Monte comme caractéristique de la concession : 
Dans les systèmes concessifs, la reprise de $\mathrm{P}$ dans la si $\mathrm{P}$ est suivie de l'assertion en $\mathrm{Q}$ d'un argument qui vient contrarier l'inférence que l'allocutaire aurait été en droit de former à partir du contenu de $\mathrm{P}$. Ce cas est très fréquent et se manifeste notamment par le nombre très élevé d'énoncés Q négatifs, portant en creux l'énoncé positif que le lecteur aurait pu inférer à partir de P. (Monte, 2009, p. 112-113)

Pourtant, elle est peut-être plutôt caractéristique pour la concession en général que pour si en emploi concessif, car les occurrences prédictives de si analysées montrent même une fréquence plus élevée de présence de négation dans Q que ne le font les cas de si concessif. Dans $12 \%$ des occurrences analysées de si en emploi conditionnel-prédictif, il y a une négation présente dans $\mathrm{Q}$, tandis que tel n'est le cas que dans $8 \%$ des occurrences analysées de si en emploi concessif. Avec même si, la fréquence de présence de négation dans $Q$ est plus importante, s'élevant à $21 \%$ des cas étudiés. Dans l'exemple 14, reprenant l'exemple 9, c'est ne... jamais qui apparaît en contexte concessif, suivant une subordonnée introduite par si. Dans l'exemple 15, c'est $n e \ldots n i \ldots n i$ qui figure dans la proposition suivant une subordonnée introduite par même si.

Exemple 14 : Pierre Laval a toujours détesté la guerre. Si, avec une apparente maestria, il a glissé de l'extrême gauche à la droite la plus conservatrice, il n’a jamais répudié l’idéal de sa jeunesse : le pacifisme. (Decaux, 1996, p. 295)

Exemple 15: Même si vous tuez quelqu'un, il n'y aura ni poursuite ni enquête. (Decaux, 1996, p. 278)

\section{$X$. Différence de position de $\mathbf{P}$}

Une différence entre l'emploi concessif de si et même si est la position de P par rapport à $\mathrm{Q}$, celle-ci étant plus libre dans le cas de même si; les contraintes de position sont plus fortes pour la subordonnée introduite par si dans son emploi concessif. Dans ce dernier, $\mathrm{P}$ précède en général $\mathrm{Q}$, et nous n'avons trouvé aucun cas dans nos données où $\mathrm{P}$ soit postposé à $\mathrm{Q}$. Par contre, dans la plupart des occurrences de même si, $\mathrm{P}$ est postposé à $\mathrm{Q}$, l'antéposition de $\mathrm{P}$ par rapport à $\mathrm{Q}$ n'apparaissant que dans $29 \%$ des occurrences étudiées de même si. En ce qui concerne l'emploi conditionnelprédictif de si, la postposition de $\mathrm{P}$ est presque aussi fréquente que l'antéposition, actualisée dans $56 \%$ des cas de nos données.

Cette différence de possibilité de position est liée aux différentes possibilités de marquage d'une relation concessive, puisque la relation concessive peut être logique, rectificative ou argumentative avec même si (Morel, 1996, p. 35). Selon la distinction des trois types de valeurs concessives de Morel, la position de P est un critère qui permet de les distinguer. La relation concessive argumentative implique que $\mathrm{P}$ est antéposé à $\mathrm{Q}$, ce qui n'est pas forcément le cas pour la relation concessive logique, et surtout pas 
pour la relation concessive rectificative, où $\mathrm{P}$ doit suivre $\mathrm{Q}^{5}$. Avec si, la relation concessive est forcément argumentative.

\section{Présence dans Q d'un marqueur concessif supplémentaire}

Une raison forte de considérer l'emploi concessif de si comme moins attendu que la relation concessive marquée par même si pourrait selon nous être la différence entre les deux conjonctions en présence d'un marqueur concessif supplémentaire. De fait, dans 81 occurrences de si en emploi concessif analysées, correspondant à $21 \%$ de la totalité des cas concessifs avec si étudiés, un autre marqueur, plus univoque, de la relation concessive, apparaît dans Q. Dans les exemples 16 à 19, c'est néanmoins, en tout cas, cependant et aussi qui apparaissent, mais il y a également d'autres marqueurs concessifs qui sont fréquents.

Exemple 16: La personne vieillissante s'inquiète de la façon dont elle est perçue par les autres. Elle souffre d'une impression d'abandon, de «dereliction » comme disent les spécialistes. Elle est traumatisée par la discordance entre l'image qu'elle a d'elle-même et celle qu'en ont les autres. Ce désaccord crée des tensions et parfois des crises d'autant que, si l'on a du mal à supporter l'image que les autres ont de vous, on est néanmoins influencé par elle. (Tubiana, 2003, p. 219-220)

Exemple 17: Si les pays occidentaux mènent entre eux une lutte de pouvoir économique et militaire sans merci, ils sont en tout cas d'accord sur la définition du cadre dans lequel se déroule cette bataille. (PostelVinay, 2005, p. 49)

Exemple 18: Si tous les observateurs s'accordent aujourd'hui pour dire que nos sociétés vivent un changement culturel important, ils ne partagent cependant pas tous l'hypothèse lourde que nous venons d'énoncer. (Bajoit, 2003, p. 63)

Exemple 19: La stabilité du rouble et l'expansion industrielle ont encouragé les investisseurs étrangers, notamment la France. Mais les décisions prises par Witte, si elles ont permis à l'économie russe d'avancer à grands pas ont eu aussi un coût politique. (Carrère d'Encausse, 2000, p. 202-203)

Si un tel marqueur supplémentaire concessif apparaît dans Q dans plus d'un cas sur cinq des occurrences de si en emploi concessif, tel n'est pas le cas pour même si dans nos données. Sur les 434 occurrences analysées de même si, il y en a seulement onze qui présentent un marqueur supplémentaire de la concession dans $\mathrm{Q}$, ce qui correspond à 2,5\% des cas étudiés de cette conjonction. L'exemple 20 comporte pourtant qui se trouve dans la proposition suivant la subordonnée introduite par même si.

5 À propos de la rectification et la distinction évoquée ci-dessus concernant la valeur polyphonique de $p$ et $q$ des trois types de concession, Léard (1987, p. 170) propose également que "[ []a postposition exclut aussi l'idée que l'on concède. » 
Exemple 20: Le plombier qui vient déboucher une canalisation, le serrurier qui en pleine nuit permet de rentrer chez soi lorsqu'on a égaré son trousseau de clefs, le dépanneur d'autoroute inspirent une certaine méfiance. Rares sont les consommateurs prêts à payer le prix sans suspicion à leur égard, même sils sont pourtant bien contents d'être ainsi promptement dépannés. (Rochefort, 1995, p. 49)

Dans la recherche d'une explication de cette différence entre les deux conjonctions quant aux tendances de marquage (supplémentaire) de la concession, il nous semble légitime de nous poser la question de savoir si ce marquage plus fréquent de la relation concessive, dans le cas de si que dans celui de même si, indique que si n'est pas un marqueur aussi «fort » de la concession que ne l'est même si. Cette idée de force réduite de marquage de la relation concessive de si par rapport à même si a également été proposée par Eriksson (1985, p. 75) :

Sur le plan sémantique aussi, il existe semble-t-il, une différence à noter entre si d'un côté, et même si et bien que (etc.) de l'autre, à savoir qu'avec si, la « concession », c'està-dire le fait de concéder l'insuffisance de la relation de cause à effet, est moins nettement mise en évidence qu'avec ses concurrents, si bien qu'il faut souvent « renforcer» la valeur concessive de la subordonnée en mettant dans la principale un adverbe ou une expression adverbiale pour souligner la validité de ce qui est exprimé dans celle-ci.

Un indice d'une telle différence de force de marquage de la relation concessive, liée au degré attendu de celle-ci, pourrait éventuellement être une différence de traduction de ces deux marqueurs dans d'autres langues. Dans une étude contrastive française-suédoise de l'emploi concessif de si et de même si, nous avons pu constater (Svensson, 2012, p. 112, 115) que dans la traduction du français en suédois, si en emploi concessif est parfois traduit par un marqueur ou une construction qui ne rend pas explicite la relation concessive, ou qui ne peut normalement pas figurer en contexte concessif. Si en emploi concessif est souvent (presque un cas sur cinq dans notre étude), comme dans l'exemple 21, traduit par om en suédois (Svensson, 2012, p. 115), qui seul ne peut marquer une relation concessive (Teleman et al., 1999, p. 649), mais qui, comme si, est le marqueur par défaut conditionnel-prédictif :

Exemple 21 : Si le modèle ancien d'éducation correspondait à une figure du Sujet dont j'ai dit qu'il était depuis longtemps en déclin, il n’en a pas moins porté une idée de l'éducation, ce que ne font pas les idées qui réduisent l'éducation à l'apprentissage des rôles sociaux et des normes qui les gouvernent. (Touraine, 1997, p. 327)

Om den gamla utbildningsmodellen överensstämde med en subjektsfigur om vilken jag har sagt att den sedan länge varit på tillbakagång, så innehöll den åtminstone en idé om utbildningen - vilket inte de åsikter gör som reducerar utbildningen till en inlärning av sociala roller och av de normer som styr dessa roller. (Touraine, 2002, p. 372) 
Étant donné que om en suédois n'apparait en général pas seul dans un contexte concessif, sans marqueur univoque de la concession, on pourrait dire que la valeur concessive est perdue ou du moins beaucoup plus implicite dans le texte cible suédois que dans le texte source français, et nous nous demandons si un tel choix de traduction aurait été possible si la concession avait été marquée par un marqueur plus univoque de la concession, comme même si. Il s'est effectivement avéré que même si est en général traduit par un marqueur concessif en suédois, comme par även om dans l'exemple 22 (Svensson, 2012, p. 116) :

Exemple 22: Même si beaucoup lisent leur horoscope, voire consultent un spécialiste des boules de cristal, c'est souvent par une sorte de réflexe, par un reste de curiosité ou à la recherche d'un petit frisson dans une vie monotone. (Muchembled, 2000, p. 296)

Även om många läser sitt horoskop eller rentav vänder sig till en kristallkuleskådare, sker det oftast av ren reflex för att man är lite nyfiken eller för att man söker lite spänning $\mathrm{i}$ en annars enahanda tillvaro. (Muchembled, 2002, p. 320)

Ces tendances différentes de traduction de si en emploi concessif et même si, ainsi que la différence en fréquence de la présence d'un marqueur concessif supplémentaire dans $\mathrm{Q}$, pourraient indiquer que la valeur concessive est parfois conçue comme moins pertinente ou moins univoque dans le cas de si que dans le cas de même si. Néanmoins, nous tenons à souligner qu'un autre marqueur concessif n'est pas nécessaire ; dans la plupart des cas, il n'est pas obligatoire, et si apparait dans la majorité des 390 cas de nos données où il figure en contexte concessif sans aucun autre marqueur concessif, comme dans l'exemple 23:

Exemple 23: Aux XVII e et XVIII e siècles, on vit apparaitre des « artistes vétérinaires », dits aussi « artistes » tout court, qui ne sont que des «maréchaux experts», sachant soigner les chevaux en maniant la lancette et le trocart, et sachant surtout les castrer. $S i$, peu à peu, des écoles se sont ouvertes dans la mouvance de celle fondée à Lyon en 1761, les vétérinaires ont longtemps continué à manquer cruellement de diplômes comme de compétences, et se sont d'ailleurs souvent vus remplacer par le médecin, en vertu du bon vieux principe selon lequel qui peut le plus peut le moins. (Beaucarnot, 2002, p. 95)

La relation concessive entre l'ouverture d'écoles vétérinaires et le manque continu de compétences des vétérinaires semble plutôt évidente, même sans ajout d'un marqueur concessif explicite. Nous nous demandons ce qui rend nécessaire l'ajout d'un marqueur concessif supplémentaire dans certains cas et pas dans d'autres, ou s'il y a, dans la plupart des cas, d'autres facteurs qui rendent impossible cet ajout, des facteurs qui l'empêcheraient de la même manière lors de l'emploi de même si. 


\section{Conclusion}

Nous avons pu constater que si en emploi concessif se distingue aussi bien de son emploi conditionnel-prédictif que de même si, et que certaines différences formelles entre ces emplois peuvent s'expliquer par le caractère inattendu de la valeur concessive de l'emploi de si par rapport au caractère attendu de la valeur concessive de même si. Il s'agit surtout de tendances différentes de marquage supplémentaire de la relation concessive par la présence d'un connecteur concessif dans la proposition qui suit la subordonnée introduite par si ou même si dans son emploi conditionnel-prédictif. Nous supposons que la fréquence plus élevée d'un marquage concessif supplémentaire dans le cas de si que dans le cas de même si est liée au caractère inattendu de la valeur concessive de l'emploi de si et au caractère plutôt attendu de la valeur concessive de même si.

\section{Bibliographie}

Corminbeuf, G., 2009, L'expression de l'bypothèse en français. Entre hypotaxe et parataxe, Bruxelles, Duculot.

Corminbeeuf, G., 2013, «Factualité et conditionnalité », in Norén, C. et al. (éds), Modalité, évidentialité et autres friandises langagières. Mélanges offerts à Hans Kronning à l'occasion de ses soixante ans, Berne, Peter Lang, coll. «Sciences pour la communication », p. 41-60.

Dancygier, B. et Sweetser, E., 2005, Mental Spaces in Grammar. Conditional Constructions, Cambridge, CUP.

Ducrot, O., 1984, Le dire et le dit, Paris, Minuit.

ERIKSSON, O., 1985, «L'emploi de même si dans les propositions concessives à valeur réelle », Studia Neophilologica, ${ }^{\circ} 57$, p. 69-78.

Gettrup, H. et Nølke, H., 1984, «Stratégies concessives: une étude de six adverbes français », Revue Romane, n 19 (1), p. 3-47.

KJELLmer, G., 1989, «Even If and Even Though », English Studies, n 3, p. 256-269.

Kronning, H., 1992, "Coordination et subordination. Aspects syntaxiques, sémantiques et pragmatiques », in Banys, W., Bednarczuk, L. et Logacki, K. (éds), Études de linguistique romane et slave. [Hommage à Stanislaw Karolak.], Cracovie, École normale supérieure (Universitas), p. 359-379.

Kronning, H., 2009a, "Polyphonie, constructions conditionnelles et discours rapporté », Langue française, $\mathrm{n}^{\circ} 164$, p. 97-111.

KRONNING, H., 2009b, «Constructions conditionnelles et attitude épistémique en français, en italien et en espagnol », Syntaxe et Sémantique, n 10, p. 13-32.

KRONNING, H., 2013, «Monstration, véridiction et polyphonie. Pour une théorie modale de la polyphonie », in Constantin de Chanay, H., Colas-Blaise, M. et Le Guern, O. (éds), Dire 
/ montrer. Au cour du sens, Chambéry, Éditions de l'Université de Savoie, coll. « Langages », p. 93-115.

KRONNING, H., à paraitre, «La théorie modale de la polyphonie et les constructions conditionnelles prédictives en si », Langages.

LÉARD, J.-M., 1987, « La syntaxe et la classification des conditionnelles et des concessives », Le français moderne, $\mathrm{n}^{\circ} 3 / 4$, p. 158-173.

LiNDSCHOUw, J., 2011, Étude des modes dans le système concessif en français du $16^{e}$ au 20e siècle et en espagnol moderne. Évolution, assertion et grammaticalisation, Copenhague, Museum Tusculanum Press.

MONTE, M., 2009, "Si marqueur d'altérité énonciative dans les si P extraprédicatives non conditionnelles ", Langue française, $\mathrm{n}^{\circ} 163$, p. 99-119.

MOREL, M.-A., 1996, La concession en français, Paris, Ophrys.

Mossberg, M., 2006, La relation de concession - Étude contrastive de quelques connecteurs concessifs en français et suédois, Växjö, Växjö University Press.

Mossberg, M., 2009, «Étude contrastive français-suédois des subordonnants conditionnels concessifs même si et även om », Studia Neophilologica, n81, p. 183-206.

Nølke, H, Fløttum, K. et NorÉn, C., 2004, ScaPoLine. La théorie scandinave de la polyphonie linguistique, Paris, Kimé.

PIOT, M., 2004, « La conjonction même si n’existe pas! » in Leclère, C. et al. (éds), Lexique, syntaxe et lexique-grammaire/Syntax, Lexis \& Lexicon-Grammar Papers in honour of Maurice Gross, Amsterdam, John Benjamins.

ProvôT, A., 2011, "Un invariant sémantique du connecteur si», Travaux du cercle belge de linguistique, $\mathrm{n}^{\circ}$ 6, http://webh01.ua.ac.be/linguist/SBKL/sbkl2011/pro2011.pdf.

STAGE, L., 1991, «Analyse syntaxique et sémantique de la conjonction dans les propositions factuelles », Revue romane, $\mathrm{n}^{\circ} 26(2)$, p. 163-205.

SVENSSON, M., 2010, Marqueurs corrélatifs en français et en suédois. Étude sémantico-fonctionnelle de d'une part... d'autre part, d'un côté... de l'autre et de non seulement... mais en contraste, Uppsala, Acta Universitatis Upsaliensis Studia Romanica Upsaliensia 79.

Svensson, M., 2012, «Analyse contrastive de même si et de si concessivo-adversatif et de leurs correspondants en suédois », Synergies Pays scandinaves, $n^{\circ}$, p. 105-121.

Svensson, M., 2013, «L'emploi concessivo-adversatif de si comparé à même si », in Norén, C. et al. (éds), Modalité, évidentialité et autres friandises langagières. Mélanges offerts à Hans Kronning à l'occasion de ses soixante ans, Berne, Peter Lang, coll. «Sciences pour la communication », p. 327-346.

Teleman, U. et al., 1999., Svenska Akademiens Grammatik, tome 4, Stockholm, Svenska Akademien. 
Maria Svensson

\section{Ouvrages du corpus}

BAjOIT, G., 2003, Le changement social: Approche sociologique des sociétés occidentales contemporaines, Paris, Armand Colin.

Beaucarnot, J.-L., 2002, Qui étaient nos ancêtres? - De leur histoire à la nôtre, Paris, Éditions JeanClaude Lattès.

Bourdieu, P., 1996, Sur la télévision suivi de L'emprise du journalisme, Paris, Éditions Raisons d'agir.

Carrère D’Encausse, H., 2000, La Russie inachevée, Paris, Librairie Arthème Fayard.

Decaux, A., 1996, C'était le XX'e siècle 2. La course à l'abîme, Paris, Librairie Académique Perrin.

DÉTREZ, C., 2002, La construction sociale du corps, Paris, Éditions du Seuil.

DubY, G., 1995, Dames du XIIe siècle 1. Hélö̈se, Aliénor, Iseut et quelques autres, Paris, Gallimard.

Muchembled, R., 2000, Une histoire du diable XIIe-XXe siècle, Paris, Éditions du Seuil.

Muchembled, R., 2002, Djänulens historia, Stockholm, Norstedts, trad. Riad, L.

Postel-VInAY, K., 2005, L'occident et sa bonne parole : nos représentations du monde, de l'Europe coloniale et l'Amérique hégénomique, Paris, Flammarion.

ROCHEFORT, R., 1995, La société des consommateurs, Paris, Odile Jacob.

SMITH, S., 2003, Négrologie - Pourquoi l'Afrique meurt, Paris, Calmann-Lévy.

TOuraine, A., 1997, Pourrons-nous viure ensemble? Égaux et différents, Paris, Librairie Arthème Fayard.

Touraine, A., 2002, Kan vi leva tillsammans? Jämlike och olika, Göteborg, Daidalos, trad. Öberg Lindsten, $\mathrm{K}$.

Tubiana, M., 2003, Le Bien-Vieillir : La révolution de l'âge, Paris, Éditions de Fallois.

DE VILLEPIN, D., 2002 [2001], Les Cent-Jours ou l'esprit de sacrifice, Paris, Perrin. 